[33] A. P. Molchanov and Ye S. Pyatnitskiy, "Criteria of asymptotic stability of differential and difference inclusions encountered in control theory," Syst. Control Lett., vol. 13, no. 1, pp. 59-64, 1989.

[34] E. S. Pyatnitskii and L. B. Rapoport, "Existence of periodic motions and test for absolute stability of nonlinear nonstationary systems in the threedimensional case," Autom. Remote Control, vol. 52, no. 5, pp. 648-658, 1991.

[35] R. N. Shorten, K. S. Narendra, and O. Mason, "A result on common quadratic Lyapunov functions," IEEE Trans. Autom. Control, vol. 48, no. 1, pp. 110-113, Jan. 2003.

\section{A Mixed Optimum in Symmetric Distributed Computer Systems}

Hisao Kameda, Eitan Altman, and Odile Pourtallier

\begin{abstract}
Consider the situation where, in a single network or system, several different types of atomic and nonatomic users coexist and have attained their own optima unilaterally. We call the combination of the optima a "mixed optimum." For a distributed system with identical nodes each having identical arrivals, we obtain the analytic expression of the unique mixed optimum, where mutual job forwarding among nodes may occur for some atomic users, resulting in paradoxical performance degradation.
\end{abstract}

Index Terms-Braess paradox, distributed decision, load balancing, Nash equilibrium, Wardrop equilibrium.

\section{INTRODUCTION}

Along with the ongoing research on road traffic equilibria, there has been a new interest in understanding competitive situations in telecommunications like the Internet as well as in parallel and distributed computing systems such as grids [1].

\section{A. Various Distributed Decisions}

We may have various separate objectives for distributed optimization of performance for different atomic or nonatomic players ${ }^{1}$ in a system, depending on the degree of the distribution of decisions. We describe each of them below in more details.

1) Completely Distributed Decision Scheme: Each of infinitely many nonatomic jobs (or of nonatomic users to whom a job belongs) optimizes its own cost. A situation is considered optimal from the point of view of that user (for a given behavior of the rest of the decision

Manuscript received October 18, 2006; revised May 18, 2007 and October 15, 2007. Recommended by Associate Editor Y. Paschalidis. The work of H. Kameda was supported in part by the University of Tsukuba Research Projects and in part by the Grant-in-Aid for Scientific Research of the Japan Society for the Promotion of Science. The work of E. Altman was supported in part by the EuroFgi European contract.

H. Kameda is with the Graduate School of Systems and Information Engineering, University of Tsukuba, Tsukuba Science City, Ibaraki 305-8573, Japan (e-mail:kameda@cs.tsukuba.ac.jp).

E. Altman and O. Pourtallier are with INRIA Sophia Antipolis, 06902 Sophia Antipolis, France (e-mail: eitan.altman@sophia.inria.fr; odile.pourtallier@sophia.inria.fr).

Digital Object Identifier 10.1109/TAC.2008.917653

${ }^{1} \mathrm{~A}$ player or a decision maker is called, respectively, atomic or nonatomic if and only if its decisions have a nonnegligible or negligible impact on the performance of any other players. makers) if it cannot expect any further benefit by changing its own decision. In the literature, the corresponding solution concept is referred to as an individual optimum, Wardrop equilibrium, or user optimum (see, e.g., Patriksson [2]). In this note, we call it individual optimum.

2) Intermediately Distributed Decision Scheme: Infinitely many jobs are classified into a finite number $(N(>1))$ of classes or groups, each of which has its own atomic decision maker and is regarded as one player or user. Each decision maker (or atomic player) optimizes noncooperatively its own cost.

We call it the class optimum.

Under quite general conditions, we know that (2) approaches (1) as $N$ tends to infinity [3].

We note, however, that most papers have investigated the cases where each system or network employs only one of these various schemes (see, e.g., [4]).

\section{B. Mixed Optimum}

On the other hand, there may be situations where, in a single network or system, several different types of atomic and nonatomic users competing for resources coexist. For example, the Internet can be modeled as being used at the same time by innumerable private users, each of whom would like to optimize only its own performance, and, by enterprizes, each of which would like to optimize only its own total cost. The usual road networks can be modeled as being used both by innumerable single owner drivers, each aiming at optimizing only his/her own travel time, and by truck and/or bus companies, each wishing to optimize their own total cost. Thus, a combination of several types of optima exists. We call the combination a mixed optimum. In this note, we study such a situation where atomic and nonatomic users coexist in a single system.

\section{Description of the System Investigated}

We consider a distributed computing system, such as a grid, composed of a network of servers or nodes on a local or wide-area network [1]. In particular, we consider a system where the nodes consist of the same kind of equipment (e.g., servers with the same characteristics) like a grid consisting of identical computers. We further assume that, in the system, each node has an identical arrival process. We refer to such a system as a system of symmetric nodes.

Note, in passing, that the analysis of nonsymmetric networks with an intermediately distributed decision scheme looks very difficult, and, to the best of the authors' knowledge, the results have been obtained only for a model of two-node distributed systems with linear link costs [5]. In [5] and [6], numerically, nonsymmetric cases have been examined analytically and numerically, and it was observed that the deterioration of the performance due to adding the connection to the system is the largest in the case of symmetric networks. This may, therefore, suggest that the symmetric topology that we study has some of the worst case features. Note that these results for nonsymmetric networks have been obtained for a single optimization and not for the mixed optimization.

\section{Previous Work}

The existence of a mixed optimum or, equivalently, a mixed equilibrium for general networks has already been shown, and its uniqueness for some special networks that do not include the network considered in this note has been shown [7]. We note, however, that the preliminary conference version [8] of this note has been the first work on a mixed optimum in the context of computer networks. Harker [9] and Richman and Shimkin [10] discuss mixed optima in contexts different from this note. 


\section{E. Summary of the Results}

In this note, we use the term "mixed optimum" in place of "mixed equilibrium." We obtain an analytic expression and uniqueness for a mixed optimum in a system of symmetric nodes. In spite of coexistence of different types of users, the results obtained in this combined system show that the behavior of each type of users, and thus, each different optimum appears to be mutually independent as though there were little interferences among one another. In this combined system with a mixed optimum, we also observe a paradoxical behavior in which there is mutual job forwarding among nodes, resulting in performance degradation, just as we have observed in a separate system with all atomic users seeking some particular type of class optimization [4]. We see, in this symmetric node model, however, that such paradoxical behavior may occur only with the atomic users seeking class optimization of a particular type, and that the nonatomic or atomic users seeking individual optimization or class optimization of other types are not subject to such a paradox nor are influenced by the paradoxes of other job types.

\section{MOdEL AND ASSUMPTIONS}

Section II-A characterizes the system of symmetrical nodes investigated in this note. Section II-B lists the set of optimization policies, one of which each of the classes coexisting in the system may employ, and defines the mixed optimum. Later, Section III characterizes the mixed optimum of the investigated system where all classes achieve their objectives coincidently. The Appendix presents the proof of the existence and uniqueness of the mixed optimum.

\section{A. Description of a System of Symmetric Nodes Where Different Types of Users Coexist}

1) Job Classes: We consider a system with $m$ nodes (computers or servers) connected with a communication means such as a grid [1]. The jobs that arrive at each node $i, i=1,2, \ldots, m$, are classified into $n$ types numbered $k=1,2, \ldots, n$. Consequently, we have $m n$ different job classes $R_{i k}$. We call such a class local class, or simply class. We also consider what we call global class $J_{k}$ that consists in the collection of local classes $R_{i k}$, i.e., $J_{k}=\bigcup_{i} R_{i k} . J_{k}$, thus, consists of all jobs of type $k$. For local class $R_{i k}$, all the jobs arrive at the same node $i$, whereas the arrivals of the jobs of global class $J_{k}$ are equally distributed over all nodes $i$.

2) Job Load-Balancing Ratios: Out of type- $k$ jobs arriving at node $i$, the ratio $x_{i j k}$ of jobs is forwarded upon arrival through the communication means to another node $j(\neq i)$ to be processed there. The remaining ratio $x_{i i k}=1-\sum_{j(\neq i)} x_{i j k}$ is processed at node $i$. We have $0 \leq x_{i j k} \leq 1$, for all $i, j, k$. A set of values of $\mathbf{x}_{i k}(i=1,2, \ldots, m, k=1,2, \ldots, n)$ are chosen to achieve optimization, where $\mathbf{x}_{i k}=\left(x_{i 1 k}, \ldots, x_{i m k}\right)$ is an $m$-vector and called "local-class $R_{i k}$ strategy." We define a global-class $J_{k}$ strategy as the $m m$-vector $\mathbf{x}_{k}=\left(\mathbf{x}_{1 k}, \mathbf{x}_{2 k}, \ldots, \mathbf{x}_{m k}\right)$. We will also denote by $\mathbf{x}$ the vector of strategies concerning all job classes, called strategy profile, i.e., the $m m n$-vector, $\mathbf{x}=\left(\mathbf{x}_{11}, \mathbf{x}_{12}, \ldots, \mathbf{x}_{1 n}\right.$, $\left.\mathbf{x}_{21}, \ldots, \mathbf{x}_{2 n}, \ldots, \mathbf{x}_{m 1}, \ldots, \mathbf{x}_{m n}\right)$, or $\mathbf{x}=\left(\mathbf{x}_{1}, \mathbf{x}_{2}, \ldots, \mathbf{x}_{n}\right)$. We denote the set of $\mathbf{x}$ 's that satisfy the constraints (i.e., $\sum_{l} x_{i l k}=1, x_{i j k} \geq$ 0 , for all $i, j, k)$ by $\mathbf{C}$. Note that $\mathbf{C}$ is a compact set.

3) Nodes and Job-Forwarding Networks: We assume that the average processing (service) time (without queueing delays) of a type- $k$ job at any node is $1 / \mu_{k}$, and is, in particular, node independent, which is like a grid consisting of identical computers. We further assume that jobs of type $k$ arrive at each node with node-independent rate $\phi_{k}$. We denote $\phi_{k} / \mu_{k}$ by $\rho_{k}$ and $\rho=\sum_{k} \rho_{k}$. We denote the total arrival rate to the node by $\phi\left(=\sum_{k} \phi_{k}\right)$, and without the loss of generality, we as-

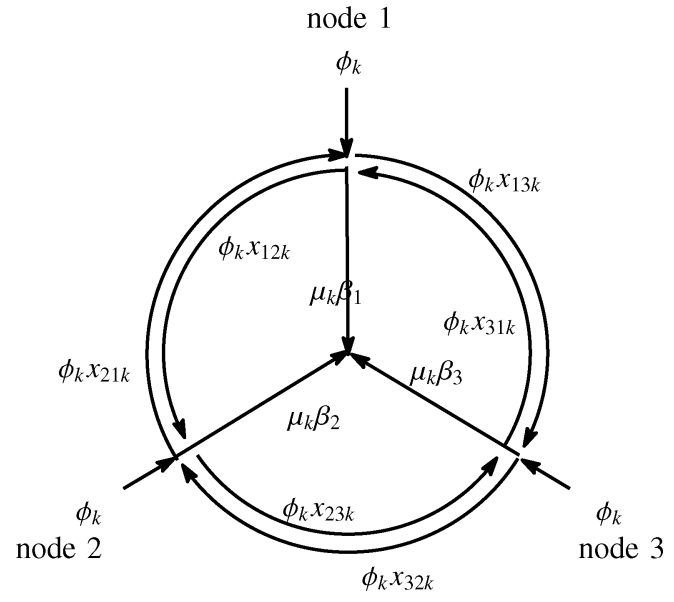

Fig. 1 Model of a system of symmetric nodes for $m=3$ for job type $k$. The symbol near each arrow denotes the rate of type- $k$ jobs that flow through the arrow. $\beta_{i}^{(k)}=\left(\phi_{k} / \mu_{k}\right) \sum_{k}\left(x_{1 i k}+x_{2 i k}+x_{3 i k}\right), i=1,2,3$.

sume a time scale such that $\phi=1$. Then, the rate $\phi_{k} x_{i j k}$ of type- $k$ jobs that arrive at node $i$ is forwarded through the communication means to node $j$, while the rate $\phi_{k} x_{i i k}$ of local-class $R_{i k}$ jobs is processed at the arrival node $i$.

For a strategy profile $\mathbf{x}$, the utilization factor $\beta_{i}$ of node $i$ is

$$
\beta_{i}=\beta_{i}(\mathbf{x})=\sum_{j, k} \rho_{k} x_{j i k} .
$$

The contribution $\beta_{i}^{(k)}$ on the utilization factor of node $i$ by the type- $k$ jobs is

$$
\beta_{i}^{(k)}=\beta_{i}^{(k)}(\mathbf{x})=\rho_{k} \sum_{j} x_{j i k}
$$

and clearly $\beta_{i}=\beta_{i}^{(1)}+\beta_{i}^{(2)}+\cdots+\beta_{i}^{(n)}$ (see Fig. 1).

Assumption П1: The expected processing (including queuing) time of a type- $k$ job that is processed at node $i$ (or the cost function at node $i$ ) is a positive, strictly increasing, strictly convex, and continuously differentiable function of $\beta_{i}$, denoted by $\mu_{k}^{-1} D\left(\beta_{i}\right)$ for all $i, k$.

Assumption $\Pi 2$ : The mean communication delay (including queuing delay) or the cost for forwarding type- $k$ jobs arriving at node $i$ to node $j$ $(i \neq j)$, denoted by $G_{i j k}(\mathbf{x})$, is a positive, nondecreasing, convex, and continuously differentiable function of $\mathbf{x} . G_{i i k}(\mathbf{x})=0$. Furthermore, each job is forwarded at most once.

In particular, we assume the following: The entire system employs as $G_{i j k}(\mathbf{x})$, for all $i, j(i \neq j), k$ one of the following functions corresponding to the type of the communication means [out of Types G-I, G-II(a), and G-II(b)] of the entire system, where $\omega_{k}$ are constants, $\sigma_{k}=\phi_{k} / \omega_{k}$, and $\underline{G}(x)$ is a nondecreasing, convex, and differentiable function of $x$ with $\underline{G}(0)=1$.

Type G-I: $G_{i j k}(\mathbf{x})=\omega_{k}^{-1} \underline{G}\left(\sigma_{k} x_{i j k}\right)$

(one dedicated line for each combination of a pair of origin and destination nodes, and a local class: i.e., $m(m-1) n$ lines in total)

Type $G$-II $(a): G_{i j k}(\mathbf{x})=\omega_{k}^{-1} \underline{G}\left(\sum_{p, q \neq p} \sigma_{k} x_{p q k}\right)$

(one bus line for each global class: i.e., $n$ bus lines in total)

Type $G-I I(b): G_{i j k}(\mathbf{x})=\omega_{k}^{-1} \underline{G}\left(\sum_{p, q(\neq p), k} \sigma_{k} x_{p q k}\right)$

(a common bus line for the entire system: i.e., 1 bus line $\}$ ).

4) Expected Job Response Times: We refer to the length of time between the instant when a job arrives at a node, and the instant when it leaves one of the nodes after all processing and communication, if 
any, are over, as the response time for the job. The expected response time of a local-class $R_{i k}$ job that arrives at node $i, T_{i k}(\mathbf{x})$, is expressed as

$$
T_{i k}(\mathbf{x})=\sum_{j} x_{i j k} T_{i j k}(\mathbf{x})
$$

where

$$
\begin{aligned}
& T_{i i k}(\mathbf{x})=\mu_{k}^{-1} D\left(\beta_{i}(\mathbf{x})\right) \\
& T_{i j k}(\mathbf{x})=\mu_{k}^{-1} D\left(\beta_{j}(\mathbf{x})\right)+G_{i j k}(\mathbf{x}), \quad \text { for } j \neq i .
\end{aligned}
$$

Using the fact that all nodes have the same arrival process, the expected response time of a global-class $J_{k}$ job is

$$
T_{k}(\mathbf{x})=\frac{1}{m} \sum_{i} T_{i k}(\mathbf{x}) .
$$

The overall expected response time of a job that arrives at the system is

$$
T(\mathbf{x})=\sum_{k} \phi_{k} T_{k}(\mathbf{x})=\frac{1}{m} \sum_{i, k} \phi_{k} T_{i k}(\mathbf{x}) .
$$

\section{B. Types of User Policies That Coexist Within the System}

Kameda and Pourtallier [4] have examined several separated systems, each of which employs only one strategy; those systems are mutually independent. Unlike in [4], we consider an entirely different situation where, within a single system, there are several decision makers that use one of different strategies discussed in Sections II-B1, II-B2, and II-B3 later for their types of jobs. That is, several types of jobs with different types of optimization decisions coexist in the same system. We denote by $\overrightarrow{\mathbf{x}}$ such a strategy profile, whereby all type- $k$ jobs achieve their own distinct performance optimization within the same system coincidentally. We call such an optimization scenario $\vec{x}$ a mixed optimum.

Definition 1: Following standard game-theoretic notation, we define $\left(\overrightarrow{\mathbf{x}}_{-(k)} ; \mathbf{x}_{k}\right)$ and $\left(\overrightarrow{\mathbf{x}}_{-(i k)} ; \mathbf{x}_{i k}\right)$, respectively, to be the $m m n$-vectors of strategies in which the elements corresponding to $\overrightarrow{\mathbf{x}}_{k}$ and $\overrightarrow{\mathbf{x}}_{i k}$, respectively, have been replaced by $\mathbf{x}_{k}$ and $\mathbf{x}_{i k}$, whereas all the other elements are the same as the remaining $m m(n-1)$ and $m(m n-1)$ elements of $\overrightarrow{\mathbf{x}}$, respectively.

We denote $\vec{\beta}_{i}=\beta_{i}(\overrightarrow{\mathbf{x}})$, which shows the utilization factor of node $i$ in the mixed optimum $\overrightarrow{\mathbf{x}}$.

We describe each of the distinct strategies coexisting in the same system in the following.

1) Individual optimization strategy for type- $k$ jobs $\mathbf{x}_{k}^{A}$ (nonatomic users): The individual optimization strategy for type- $k$ jobs (where nonatomic users seek individual optimization) is denoted by $\mathbf{x}_{k}^{A}$.

For type- $k$ jobs seeking individual optimization, the strategy of type$k$ jobs, given the strategies of the other classes, is then presented by such $\mathbf{x}_{k}^{A}$ as satisfies the following conditions for all $i$

$$
T_{i k}\left(\overrightarrow{\mathbf{x}}_{-(k)} ; \mathbf{x}_{k}^{A}\right)=\min _{j}\left\{T_{i j k}\left(\overrightarrow{\mathbf{x}}_{-(k)} ; \mathbf{x}_{k}^{A}\right)\right\} \text { and } \quad\left(\overrightarrow{\mathbf{x}}_{-(k)} ; \mathbf{x}_{k}^{A}\right) \in \mathbf{C}
$$

where, for $\left(\overrightarrow{\mathbf{x}}_{-(k)} ; \mathbf{x}_{k}^{A}\right)$, see Definition 1 .

In the individual optimization strategy for type- $k$ jobs, we consider that each job of type $k$ chooses the node to be processed in order to obtain its minimal cost, i.e., the minimal expected response time for itself, given the decisions on other jobs. Thus, for global-class $J_{k}$ (consisting of local-class $R_{i k}$ for all $i$ ), there exist infinitely many decision makers. The resulting optimal ratio of jobs of local class $R_{i k}$ that choose the node $j$ to be processed is denoted by $x_{i j k}^{A}$. Thus, for type- $k$ jobs, nonatomic users seek individual optimization that results in the individually optimal strategy profile of type- $k$ jobs denoted by $\mathbf{x}_{k}^{A}$. The relation (8) means the following: if nonatomic users seek the individual optimum for type- $k$ jobs, the cost of paths used by type- $k$ jobs are identical, are given by $T_{i k}\left(\overrightarrow{\mathbf{x}}_{-(k)} ; \mathbf{x}_{k}^{A}\right)$ and are not larger than the cost of paths that are not used by type- $k$ jobs.

2) Local-class optimization strategy for type- $k$ jobs $\mathbf{x}_{k}^{B I}$ (some atomic user in the system): The local-class optimal strategy for decision maker $(i k)$, or equivalently, local-class job $R_{i k}$ is denoted by the $m$-vector $\mathbf{x}_{i k}^{B I}=\left(x_{i 1 k}^{B I}, x_{i 2 k}^{B I}, \ldots, x_{i m k}^{B I}\right)$. We assume that the local classes $R_{i k}$ for all $i$ with the identical $k$ are subject to localclass optimization strategy coincidentally, and then, an optimal strategy profile for type- $k$ jobs, that we denote by $\mathbf{x}_{k}^{B I}$, is the $m m$-vector $\left(\mathbf{x}_{1 k}^{B I}, \mathbf{x}_{2 k}^{B I}, \ldots, \mathbf{x}_{m k}^{B I}\right)$.

For type- $k$ jobs seeking local-class optimization, the strategy of type- $k$ jobs, given the strategies of the other classes, is presented by such $\mathbf{x}_{k}^{B I}$ as satisfies the following for all $i$

$$
\begin{aligned}
& T_{i k}\left(\overrightarrow{\mathbf{x}}_{-(i k)} ; \mathbf{x}_{i k}^{B I}\right)=\min T_{i k}\left(\overrightarrow{\mathbf{x}}_{-(i k)} ; \mathbf{x}_{i k}\right) \\
& \text { with respect to } \mathbf{x}_{i k} \text { such that }\left(\overrightarrow{\mathbf{x}}_{-(i k)} ; \mathbf{x}_{i k}\right) \in \mathbf{C}
\end{aligned}
$$

where for $\left(\overrightarrow{\mathbf{x}}_{-(i k)} ; \mathbf{x}_{i k}^{B I}\right)$ and $\left(\overrightarrow{\mathbf{x}}_{-(i k)} ; \mathbf{x}_{i k}\right)$, see Definition 1.

In the local-class optimization strategy for type- $k$ jobs, each local class $R_{i k}$ has its own nonatomic decision maker $(i k)$. The amount of forwarding for local-class $R_{i k}$ jobs is chosen by the corresponding decision maker $(i k)$ in order to obtain the minimal cost, i.e., the expected response time averaged over all the local-class $R_{i k}$ jobs, given the decisions on other job classes.

3) Global-class optimization strategy $\mathbf{x}_{k}^{B I I}$ (some other atomic user): The global-class optimal strategy for decision maker $k$ is denoted by an $m m$-vector

$$
\mathbf{x}_{k}^{B I I}=\left(\mathbf{x}_{1 k}^{B I I}, \mathbf{x}_{2 k}^{B I I}, \ldots, \mathbf{x}_{m k}^{B I I}\right)
$$

For type- $k$ jobs seeking global-class optimization, the strategy of type$k$ jobs, given the strategies of the other classes, is presented by such $\mathbf{x}_{k}^{B I I}$ as satisfies the following

$$
\begin{aligned}
& T_{k}\left(\overrightarrow{\mathbf{x}}_{-(k)} ; \mathbf{x}_{k}^{B I I}\right)=\min T_{k}\left(\overrightarrow{\mathbf{x}}_{-(k)} ; \mathbf{x}_{k}\right) \\
& \text { with respect to } \mathbf{x}_{k} \text { such that }\left(\overrightarrow{\mathbf{x}}_{-(k)} ; \mathbf{x}_{k}\right) \in \mathbf{C}
\end{aligned}
$$

where for $\left(\overrightarrow{\mathbf{x}}_{-(k)} ; \mathbf{x}_{k}^{B I I}\right)$ and $\left(\overrightarrow{\mathbf{x}}_{-(k)} ; \mathbf{x}_{k}\right)$, see Definition 1 .

In the global-class optimization strategy for type- $k$ jobs, jobs of local classes $R_{i k}$ for all $i$ are united into one global class $J_{k}$ that has a single atomic decision maker $(k)$. Each decision maker $(k)$ of global class $J_{k}$ chooses the amount of job forwarding for the $m$ local classes, $R_{1 k}, R_{2 k}, \ldots, R_{m k}$ in order to obtain the minimal cost, i.e., the expected response time averaged over all the global class $J_{k}$ jobs, given the decisions on other job types.

1) Mixed Optimization $\overrightarrow{\mathbf{x}}$ : Now, we recall the definition of the mixed optimum given in the Introduction. That is, a strategy profile $\overrightarrow{\mathbf{x}}$ is called a mixed optimum if, for all $k$, its $k$ th component is one of different optima for type- $k$ jobs given $\overrightarrow{\mathbf{x}}_{-(k)}$. In other words, if type- $k$ jobs seek individual optimization, then $\overrightarrow{\mathbf{x}}_{k}=\mathbf{x}_{k}^{A}$, where $\mathbf{x}_{k}^{A}$ is given in (8), if type- $k$ jobs seek local-class optimization, then $\overrightarrow{\mathbf{x}}_{k}=\mathbf{x}_{k}^{B I}$, where $\mathbf{x}_{k}^{B I}$ is given in (9), and if type- $k$ jobs seek global-class optimization then $\overrightarrow{\mathbf{x}}_{k}=\mathbf{x}_{k}^{B I I}$, where $\mathbf{x}_{k}^{B I I}$ is given in eq. (10).

We denote the numbers of job types seeking the individual optimization, the local-class optimization, and the global-class optimization by $n_{A}, n_{B-I}$, and $n_{B-I I}$, respectively. Then, $n_{A}+n_{B-I}+n_{B-I I}=n$. We denote $\Gamma_{k}=\rho_{k}^{2} \sigma_{k}^{-1}$. 


\section{SOlution AND ITS UNIQUENESS OF A MiXed OPTIMUM}

Now, we have the main result. Based on the earlier characterizations given in Section II, we show that the solution $\overrightarrow{\mathbf{x}}$ for all job types is unique and given as follows. We define

$$
g_{i j k}^{B I}(\mathbf{x})=\frac{\partial}{\partial x_{i j k}}\left\{\phi_{k} \sum_{p \neq i} x_{i p k} G_{i p k}(\mathbf{x})\right\} .
$$

\section{A. Unique Mixed Optimum $\overrightarrow{\mathbf{x}}$ for the System Investigated}

1) The solution $\mathbf{x}_{k}^{A}$ for type $k$ with individual optimization is unique and given as follows:

$$
x_{i j k}^{A}=0 \text { and } x_{i i k}^{A}=1, \quad \text { for all } i, j(\neq i) .
$$

The mean response time for type $k$ with individual optimization is

$$
T_{k}(\overrightarrow{\mathbf{x}})=T_{i k}(\overrightarrow{\mathbf{x}})=\mu_{k}^{-1} D(\rho), \quad \text { for all } i .
$$

2) The solution $\mathbf{x}_{k}^{B I}$ for type- $k$ jobs with local-class optimization is unique and is given as follows: If the entire system employs Types G-I or G-II(a) communication means:

a) for local class $R_{i k}$ such that $\rho_{k}^{2} D^{\prime}(\rho) \leq g_{k}^{B I}(0)=\sigma_{k}$, i.e., $\Gamma_{k} D^{\prime}(\rho) \leq 1$

$$
x_{i j k}^{B I}=0 \text { and } x_{i i k}^{B I}=1, \quad \text { for all } i, j(\neq i) .
$$

The mean response time for local class $R_{i k}$ jobs with local-class optimization, such that $\Gamma_{k} D^{\prime}(\rho) \leq 1$, is given by (13);

b) for local class $R_{i k}$ such that $\rho_{k}^{2} D^{\prime}(\rho)>g_{k}^{B I}(0)=\sigma_{k}$, i.e., $\Gamma_{k} D^{\prime}(\rho)>1$

$$
x_{i j k}^{B I}=x_{k}^{B I}, \quad \text { for all } i, j(\neq i)
$$

where $x_{k}^{B I}$ is the unique solution of

$$
\begin{aligned}
& \rho_{k}^{2}\left(1-m x_{k}^{B I}\right) D^{\prime}(\rho)=g_{k}^{B I}\left(x_{k}^{B I}\right)=\sigma_{k}\left[\underline{G}\left(m(m-1) \sigma_{k} x_{k}^{B I}\right)\right. \\
& \left.\quad+\sigma_{k}(m-1) x_{k}^{B I} \underline{G}^{\prime}\left(m(m-1) \sigma_{k} x_{k}^{B I}\right)\right] .
\end{aligned}
$$

The mean response time for local-class $R_{i k}$ jobs with local-class optimization, such that $\Gamma_{k} D^{\prime}(\rho)>1$, is, for all $i$

$$
T_{k}(\overrightarrow{\mathbf{x}})=T_{i k}(\overrightarrow{\mathbf{x}})=\mu_{k}^{-1} D(\rho)+(m-1) x_{k}^{B I} G_{k}(\overrightarrow{\mathbf{x}}) .
$$

If the entire system employs Type G-II(b) communication means:

The solution is given as follows:

$$
x_{i j k}^{B I}=x_{k}^{B I}, \text { for all } i, j(\neq i)
$$

where $x_{k}^{B I}$ is given as in the following. We first change the numbering of $k$, such that $\Gamma_{1} \geq \Gamma_{2} \geq \cdots \geq \Gamma_{k} \geq \cdots \geq \Gamma_{n^{\prime}}$, where $n^{\prime}$ is the number of job types that seek the local-class optimization, i.e., $n^{\prime}=n_{B-I}$. The following three situations can occur: We can find $K$ such that

$$
\begin{aligned}
& \quad \Gamma_{K} D^{\prime}(\rho)>1 \text { and } \Gamma_{K+1} D^{\prime}(\rho) \leq 1 \\
& \text { or } \left.\Gamma_{n^{\prime}} D^{\prime}(\rho)>1 \quad \text { (i.e., } K=n^{\prime}\right) \\
& \text { or } \Gamma_{1} D^{\prime}(\rho) \leq 1
\end{aligned}
$$

When (21) holds, we have a unique solution of $x_{k}^{B I}=0$ for all $k \leq n^{\prime}$. When (19) or (20) holds, we can find a unique solution as follows. Let us define the function $F_{k}(X)$ as

$$
F_{k}(X)=\left\{\sum_{l=1}^{k} \frac{\sigma_{l}\left[\Gamma_{l} D^{\prime}(\rho)-\underline{G}(X)\right]}{m \Gamma_{k} D^{\prime}(\rho)+(m-1) \sigma_{l} \underline{G^{\prime}}(X)}\right\}-\frac{X}{m(m-1)} .
$$

We obtain the largest $k=k^{B I} \leq K$ and $X=X_{k^{B I}}^{B I}(>0)$ that satisfies $F_{k B I}\left(X_{k B I}^{B I}\right)=0$ and $\sigma_{k B I}\left[\Gamma_{k B I} D^{\prime}(\rho)-\underline{G}\left(X_{k B I}^{B I}\right)\right]>0$. Then, by using

$$
\begin{aligned}
\sigma_{k} & {\left[\Gamma_{k} D^{\prime}(\rho)-\underline{G}\left(X_{k B I}^{B I}\right)\right] } \\
& =\sigma_{k} x_{k}^{B I}\left[m \Gamma_{k} D^{\prime}(\rho)+(m-1) \sigma_{k} \underline{G^{\prime}}\left(X_{k}^{B I}\right)\right]
\end{aligned}
$$

for all $k$, for $k=1,2, \ldots, k^{B I}$, we can obtain the unique set of values such that $x_{k}^{B I}>0, k=1,2, \ldots, k^{B I}$, and that $x_{k^{B I}+1}^{B I}=x_{k^{B I}+2}^{B I}=$ $\cdots=x_{n^{\prime}}^{B I}=0$ that satisfies the previous relation, which is a unique solution.

The mean response time for local class $R_{i k}$ jobs with local-class optimization is given by (17), for all $i$.

3) The solution $\mathbf{x}_{k}^{B I I}$ for type- $k$ jobs with global-class optimization is unique and is given as follows:

$$
x_{i j k}^{B I I}=0 \quad \text { and } \quad x_{i i k}^{B I I}=1, \quad \text { for all } i, j(\neq i) .
$$

The mean response time for type- $k$ jobs with global-class optimization is given by (13).

The aforementioned can be confirmed as follows.

Theorem 3.1: Consider a system of symmetric nodes with several coexisting types of jobs where each type optimizes according to one of the strategies 1), 2), or 3) discussed earlier. Then, for the system, there exists a unique mixed optimum $\overrightarrow{\mathbf{x}}$, which is given before.

Proof: See the appendix.

Remark 3.1: In the earlier mixed-optimum solution $\overrightarrow{\mathrm{x}}$, we see that although various types of players or classes exist together in a single system considered, the behavior of each class or player in the (unique) mixed optimum $\vec{x}$ appears to be independent of one another. We also see that Braess-like paradoxical performance degradation occurs only for the type of jobs seeking local-class optimization, and whether or not the Braess-like paradox occurs depends on the system load $\rho\left(=\sum_{k} \rho_{k}\right)$ and the value of $\Gamma_{k}\left(=\rho_{k}^{2} / v_{k}=\phi_{k} \omega_{k} / \mu_{k}^{2}\right)$ for each local-class $R_{i k}$, where $\phi_{k}$ is the arrival rate, $\mu_{k}^{-1}$ is the processing time requirement, and $\omega_{k}^{-1}$ is the communication time requirement for local-class $R_{i k}$. The types of jobs seeking individual or global-class optimization are not influenced by such performance degradation.

We discuss next the possible implications of our results in the context of road traffic.

Remark 3.2: In road traffic, some of the drivers choose routes so as to minimize their individual delay (individual optimization), while others are drivers of companies that provide drivers with directions on how to choose routes to minimize the average delay of their drivers (local-class optimization). A surprising implication of our results is that the performance of independent drivers may dominate that of drivers employed by companies where a company is assumed to decide on the route to be taken by each of the cars that belongs to it. We call such drivers "company drivers." Indeed, consider a road network with three nodes: two origins $(i, j(\neq i)=1,2)$ and one common destination $d$, as a very simple example of the networks we have studied. Some drivers arrive at a node $i$ and take a direct route to $d$. The remaining drivers arrive at node $i$ and reach node $d$ through an alternate route $i j d$. Assume that there are local companies $R_{i k}$, each of which gives directions to its drivers. It can be seen from our results that at the unique mixed optimum, the decisions of such companies may be inefficient since they may involve mutual forwarding between nodes 1 and 2 . We conclude that this situation may discourage company drivers and that companies should take into consideration the possible negative impact of their directions to optimize the average delay of their drivers only. 


\section{CONCLUding REMARKS}

In this note, we have examined a model consisting of identical nodes with identical arrivals to all nodes where forwarding of jobs to the other nodes through communication means with nonzero delays may clearly lead to performance degradation. We have considered mixed optimization where each of the coexisting job types seeks a distinct level of distributed optimization. We have computed explicitly a mixed optimum. We have established the uniqueness of a mixed optimum. We have observed a paradoxical behavior in which in a mixed optimum there is mutual job forwarding among nodes.

It has been quite hard to extend the proofs to allow more general assumptions. It has been also difficult for us to analyze asymmetric models. These are future open problems.

\section{APPENDIX A: A PROOF OF THEOREM 3.1}

We show that, under the assumption that there exists a mixed optimum, a unique solution $\overrightarrow{\mathbf{x}}$ is given as shown in the following lemmas $1,2,3$, and 4 . On the other hand, we can see that solution $\vec{x}$ given by these lemmas and mentioned in this theorem satisfies the definition of a mixed optimum. We, therefore, see that a mixed optimum exists and is uniquely given as the $\overrightarrow{\mathrm{x}}$ mentioned before.

Lemma 1: Consider a network with several types of jobs, where each type optimizes according to either one of the strategies 1), 2), or 3 ). If there exists a mixed optimum in the network, then, in the mixed optimum $\overrightarrow{\mathbf{x}}$, we must have $\vec{\beta}_{i}=\rho$, for all $i$.

Proof: This can be shown by augmenting the part (1) of the proof given on pp. 419-421 of [4], see [8].

Lemma 2: If there exists a mixed optimum for the network, then, in the mixed optimum $\overrightarrow{\mathbf{x}}$, the solution $\mathbf{x}_{k}^{A}$ for type $k$ with individual optimization is unique and given by (12). The mean response time is given by (13).

Proof: We define

$$
t_{i j k}^{A}(\mathbf{x})=\phi_{k} T_{i j k}(\mathbf{x}) .
$$

For all $i, j$, we have

$$
\begin{gathered}
t_{i j k}^{A}\left(\overrightarrow{\mathbf{x}}_{-(k)} ; \mathbf{x}_{k}^{A}\right)=\alpha_{i k}^{A}, \quad x_{i j k}^{A}>0 \\
t_{i j k}^{A}\left(\overrightarrow{\mathbf{x}}_{-(k)} ; \mathbf{x}_{k}^{A}\right) \geq \alpha_{i k}^{A}, \quad x_{i j k}^{A}=0 \\
\sum_{j^{\prime}} x_{i j^{\prime} k}^{A}=1
\end{gathered}
$$

where $\alpha_{i k}^{A}=\min _{j^{\prime}}\left\{\phi_{k} D\left(\beta_{j^{\prime}}\left(\overrightarrow{\mathbf{x}}_{-(k)} ; \mathbf{x}_{k}^{A}\right)\right)\right\}$.

If there exists a mixed optimum for the network, then, in the mixed optimum $\overrightarrow{\mathbf{x}}$, the solution $\mathbf{x}_{k}^{A}$ for type $k$ with individual optimization exists. Then, the set of relations (26) must hold, and it is satisfied if and only if $x_{i j k}^{A}=0$ for all $i, j(\neq i)$.

Lemma 3: If there exists a mixed optimum for the network, then, in the mixed optimum $\overrightarrow{\mathbf{x}}$, the solution $\mathbf{x}_{k}^{B I}$ for type- $k$ jobs with local-class optimization is unique and is given as follows.

If the entire system employs Types G-I or G-II(a) communication means:

1) for local class $R_{i k}$ such that $\rho_{k}^{2} D^{\prime}(\rho) \leq g_{k}^{B I}(0)=\sigma_{k}$, i.e., $\Gamma_{k} D^{\prime}(\rho) \leq 1, \boldsymbol{x}_{k}^{B I}$ is given by (14). The mean response time is given by (13);
2) for local class $R_{i k}$ such that $\rho_{k}^{2} D^{\prime}(\rho)>g_{k}^{B I}(0)=\sigma_{k}$, i.e., $\Gamma_{k} D^{\prime}(\rho)>1$, the solution $\mathbf{x}_{k}^{B I}$ is given by (15). The mean response time is given by (17).

If the entire system employs Type G-II(b) communication means:

The solution $\mathbf{x}_{k}^{B I}$ is given by (18). The mean response time is given by (17).

Proof: 1) By Lemma 1, we have shown that $\vec{\beta}_{j}=\vec{\beta}_{j^{\prime}}$ for every pair of $\left(j, j^{\prime}\right)$, and consequently, $\vec{\beta}_{i}=\rho$ for all $i$.

2) The rest of the proof can be shown by the logic similar to that in parts (2) and (3) of the proof starting on p. 418 of [4], where $n$ in part (3) is to be replaced by $n_{B-I}$ and, where, for any variable, say, $y$, in part (2), $\tilde{y}$ is to be replaced by $y^{B I}$.

Lemma 4: If there exists a mixed optimum for the network, then, in the mixed optimum $\overrightarrow{\mathbf{x}}$, the solution $\mathbf{x}_{k}^{B I I}$ for type- $k$ jobs with globalclass optimization is unique and is given by (24). The mean response time is given by (13).

Proof: 1) By Lemma 1, we have shown that $\vec{\beta}_{j}=\vec{\beta}_{j^{\prime}}$ for every pair of $\left(j, j^{\prime}\right)$, and consequently, $\vec{\beta}_{i}=\rho$ for all $i$.

2) The rest of the proof can be shown by the logic similar to that in parts (2) and (3) of the proof starting on p. 424 of [4], where, for any variable, say, $y$, in part (2), $\check{y}$ is to be replaced by $y^{B I I}$.

\section{ACKNOWLEDGMENT}

The authors would like to thank Prof. C. Altman for improving the presentation of this note.

\section{REFERENCES}

[1] I. Foster and C. Kesselman, Eds., The Grid: Blueprint for a New Computing Infrastructure. San Mateo, CA: Morgen Kaufmann, 1998.

[2] M. Patriksson, The Traffic Assignment Problem-Models and Methods. Utrecht, The Netherlands: VSP, 1994.

[3] A. Haurie and P. Marcotte, "On the relationship between NashCournot and Wardrop equilibria," Networks, vol. 15, pp. 295-308, 1985.

[4] H. Kameda and O. Pourtallier, "Paradoxes in distributed decisions on optimal load balancing for networks of homogeneous computers," $J$. ACM, vol. 49, no. 3, pp. 407-433, 2002.

[5] H. Kameda, M. Ohta, and Y. Hosokawa, "Effects of symmetry on paradoxical cost degradation in a Nash non-cooperative network system," presented at the IFAC Symp. Modeling Control Econ. Syst. (SME 2001), Klagenfurt, Austria.

[6] S. F. El-Zoghdy, H. Kameda, and J. Li, "Numerical studies on a paradox for non-cooperative static load balancing in distributed computer systems," Comput. Operations Res., vol. 33, pp. 345-355, 2006.

[7] T. Boulogne, E. Altman, H. Kameda, and O. Pourtallier, "Mixed equilibrium (ME) for multiclass routing games," IEEE Trans. Automat. Control, Special Issue Control Issues Telecommun. Netw., vol. 47, no. 6, pp. 903916, Jun. 2002.

[8] H. Kameda, E. Altman, and O. Pourtallier, "Paradoxes on mixed optima in symmetric distributed computer systems," in Proc. 9th Int. Symp. Dyn. Games Appl., Adelaide, Autralia, Aug.2000, pp. 309-324.

[9] P. Harker, "Multiple equilibrium behaviors on networks," Transport. Res $B$, vol. 22, pp. 39-46, 1988.

[10] O. Richman and N. Shimkin, "Topological uniqueness of the Nash equilibrium for atomic selfish routing," Math. Operations Res., vol. 32, no. 6, pp. 215-232, Feb. 2007. 